\title{
Über die Wirkung des Isokarboxazid, Reserpin und Prochlorperazin auf die Menge des Zuckers, der Brenztraubensäure und des Cholesterins im Kaninchenblut
}

\author{
von \\ Masafumi KOBAYASHI*, Yutaka ASAKURA** \\ und Yoshiko WAKAMATSU***
}

Schon war es von Kobayashi [1], eine Mitalbeiter der besagte Wirkung, mitgeteilt, dass die Tranquillizer aus den Phenothiazinderivaten die Karies der Rattenmolaren verstärkten. Noch berichtet ASAKURA [2], derselbe Albeiter, dass Reserpin, Tranquillizer aus dem Rauwolfia Alkaloid, als die Phenothiazinderivaten mehr wirkt. Neulich aufs nue tragtet er die Fähigkeit von Isokarboxazid, Monaminoxydase-Hemmer (Mo-H) solcher Tatsache durch Reserpin zu schwächen, nach [3].

Von Brodie [4,5] ist die Sedation mit Reserpin aus Freisetzungen der Serotonin in das Gehirn entspringt, aber die Vorbehandlung von Mo-H (Iproniazid) schwächt seine Wirkung oder verursacht die Erregung. Wenn freier Serotonin wieviel Stunden stück für stück im Zentrum produziert, verursacht es die Sedation, jodoch solcher pharmakologische Antagonismus aus die übergrosse Zunahme des Serotonin an Quantität während einer kurzen Stunden besteht.

Isokarboxazid, der von ASAKURA gebrauchene Mo-H, bezeigt nicht den autonomische kleinliche Effekt mit subkutaner Injektion. Demnach folgerten wir es, dass die Hemmung von Isokarboxazid, der durch Reserpin hervorgerufene Verstärkung der Karies, zu Quantität des Serotonin Beziehungen hat.

Anderseits ist die Zunahme an Quantität des Blutzucker mit Reserpin für unserer Mechanismus gedacht. Es ist von NIINOH [6] und WaKamatsu [7] unseres Institutes mitgeteilt worden, dass die Tranquillizer, besonders Reserpin, Blutzucker sowie Brenztraubensäure der Ratten vermehrten, jedoch sie Isokarboxazid zu kombinieren gefallen waren.

Diesmal, mit dem Zweck, den Wirkungsmechanismus solcher Tatsache auch zu verfolgen, verabreichten wir den Kaninchen statt die Ratten die gleichen Mitteln, und fügten als gemessenen Gegenständ Cholesterin mit den vorhergehenden Stoffen hinzu.

\section{Methodik}

Das experimentelle Tiere: Die Kaninchen (male) im Gewicht zwischen 1.5-2.0 Kg am Anfang der Züchten.

Der Zeit auf Züchten : 30 Tagen.

Das Heilmittel :

*小林 雅文, **朝倉 裕, ***若松 桂子： Pharmakologische Institut, Zahnärztliche Fakultät der Nihon Universität zu Tokyo (Leiter: Prof. Dr. Mikio Sato) 
1) Reserpin (Reserpin, Dai-Nihon-Seiyaku) $1 \mathrm{mg} / \mathrm{ml}$

2) Prochlorperazin (Novamin, Shionogi-Seiyaku) $5 \mathrm{mg} / \mathrm{ml}$

3) Isokarboxazid (Enerzer, Takeda-Yakuhin) $2 \mathrm{mg} / \mathrm{ml}$ (Lösungsmittel für Enerzer : Propyrenglykol)

Täglich machten die Autor subkutane Injektion dieser Arzneien den Kaninchen auf dem fastsetzten Zeit.

Das Dosis für Injektion pro Tag: Reserpin $0.4 \mathrm{mg} / \mathrm{kg}$, Prochlorperazin $4 \mathrm{mg} / \mathrm{kg}$, Isokarboxazid $2 \mathrm{mg} / \mathrm{kg}$.

Die quantitative Analyse des Stoffs :

1) Blutzucker - Hagedorn-Jensen'sche Methode [8].

2) Brenztraubensäure - Friedemann-Haugen'sche Methode (Dinitrophenyl Hydrazin Methode) [9].

3) Cholesterin - Blorr'sche Methode (Azetische Anhydride Methode)[10].

Jeder war von Blut extrahiert, das aus Vena auricularis an den Tieren unter der Ruhe gewonnen war. Die Analysen der Stoff wurden je ein Mal vor und nach die Zeit der Arzneidarreichung getan, die so die Quantitäten vor und nach vergleicht waren.

Die Zusammensetzung der Gruppe :

1) $\mathrm{Mo}-\mathrm{H}$ (Isokarboxazid) Gruppe - nur Mo-H administriert.

2) $\mathrm{Mo}-\mathrm{H}+$ Reserpin Gruppe - mit Mo-H, Reserpin administriert.

3) $\mathrm{Mo}-\mathrm{H}+$ Prochlorperazin Gruppe - mit $\mathrm{Mo}-\mathrm{H}$, Prochlorperazin administriert.

\section{Ergebnisse}

1) Der Einfluss auf Blutzucker.

Mo-H Gruppe (Tab. 1): Die durchschnittliche Grosse $90.76 \mathrm{mg} / \mathrm{dl}$ vor den Zeit der Arzneidarreichung war höher als die Grosse $72.94 \mathrm{mg} / \mathrm{dl}$ nach dem $(0.10>\mathrm{p}>0.05)$.

$\mathrm{Mo}-\mathrm{H}+$ Reserpin Gruppe (Tab. 2): Die Differenz vor und nach dem Zeit war sehr minimal $(p>0.50)$.

TABELLE 1

Blutzucker

$(\mathrm{mg} / \mathrm{dl})$

\begin{tabular}{|c|c|c|c|}
\hline \multicolumn{4}{|c|}{ Mo-H Gruppe } \\
\hline Tier Nr. & VOR* & $\mathrm{NACH}^{* *}$ & $\mathrm{NACH}-\mathrm{VOR}$ \\
\hline 1 & 77.17 & 48.49 & -28.68 \\
\hline 2 & 77.17 & 138.16 & 60.99 \\
\hline 3 & 106.20 & 89.99 & -16.21 \\
\hline 4 & 96.64 & 48.49 & -48.15 \\
\hline 5 & 96.64 & 39.57 & -57.07 \\
\hline Mittelwerte & 90.76 & 72.94 & $-17.82^{+}$ \\
\hline $\begin{array}{c}* \quad \text { vor admin } \\
* * \text { nach admin }\end{array}$ & $\begin{array}{l}\text { Zeit. } \\
\text { Zeit. }\end{array}$ & & $+0.10>\mathrm{P}>0.05$ \\
\hline
\end{tabular}




\section{TABELLE 2 \\ Blutzucker}

$(\mathrm{mg} / \mathrm{dl})$

\begin{tabular}{|c|c|c|c|}
\hline \multicolumn{4}{|c|}{ Mo-H+Reserpin Gruppe } \\
\hline Tier Nr. & VOR & $\mathrm{NACH}$ & $\mathrm{NACH}-\mathrm{VOR}$ \\
\hline 1 & 94.27 & 91.95 & -2.32 \\
\hline 2 & 113.63 & 87.11 & -26.52 \\
\hline 3 & 101.63 & 101.63 & 0.00 \\
\hline 4 & 106.47 & 108.99 & 1.46 \\
\hline 5 & 89.44 & 91.95 & 2.51 \\
\hline Mittelwerte & 101.08 & 96.34 & $\begin{array}{l}-14.77^{--} \\
---P>0.50\end{array}$ \\
\hline
\end{tabular}

TABELLE 3

Blutzucker

$(\mathrm{mg} / \mathrm{dl})$

\begin{tabular}{|c|c|c|c|}
\hline \multicolumn{4}{|c|}{$\mathrm{Mo}-\mathrm{H}+$ Prochlorperazin Gruppe } \\
\hline Tier Nr. & VOR & $\mathrm{NACH}$ & $\mathrm{NACH}-\mathrm{VOR}$ \\
\hline 1 & 95.23 & 32.37 & -62.86 \\
\hline 2 & 70.80 & 116.37 & 45.57 \\
\hline 3 & 90.27 & 46.07 & -44.20 \\
\hline 4 & 93.10 & 130.90 & 37.80 \\
\hline 5 & 87.44 & 133.32 & 45.88 \\
\hline Mittelwerte & 87.37 & 91.81 & $\begin{array}{r}4.44^{---} \\
---P>0.50\end{array}$ \\
\hline
\end{tabular}

Mo-H+Prochlorperazin Gruppe (Tab. 3): Nicht die Differenz ( $\mathrm{p}>0.50)$.

2) Der Einfluss auf Brenztraubensäure.

Mo-H Gruppe (Tab. 4) : Gegen das Resultat auf Blutzucker war höher die Grosse $(2.16 \mathrm{mg} / \mathrm{dl})$ nach den Zeit als $(1.94 \mathrm{mg} / \mathrm{dl})$ vor den $(0.10>\mathrm{p}>0.05)$.

Mo-H+Reserpin Gruppe (Tab. 5): Das Resultat deren Gruppe hatte gleiche Tendenz mit der Mo-H Gruppe (vor : $2.52 \mathrm{mg} / \mathrm{dl}$, nach : $4.48 \mathrm{mg} / \mathrm{dl}, \mathrm{p}>0.001$ ). Kurzum, Zunahme der Brenztraubensäure durch Isokarboxazid war nicht mit Reserpin inhibiert.

Mo-H + Prochlorperazin Gruppe (Tab. 6) : Auch die Gruppe hattet gleiche Tendenz mit der Mo-H Gruppe (vor : $2.24 \mathrm{mg} / \mathrm{dl}$, nach : $2.64 \mathrm{mg} / \mathrm{dl}, 0.025>\mathrm{p}>0.01$ ). Kurzum, Prochlorperazin inhibiertet nicht Zunahme der Brenztraubensäure. 
TABELLE 4

Brenztraubensäure in Blut

(mg/dl)

\begin{tabular}{|c|c|c|c|}
\hline \multicolumn{4}{|c|}{ Mo-H Gruppe } \\
\hline Tier Nr. & VOR & $\mathrm{NACH}$ & $\mathrm{NACH}-\mathrm{VOR}$ \\
\hline 1 & 1.70 & 2.12 & 0.42 \\
\hline 2 & 2.08 & 2.12 & 0.04 \\
\hline 3 & 2.08 & 2.12 & 0.04 \\
\hline 4 & 1.70 & 2.18 & 0.48 \\
\hline 5 & 2.15 & 2.24 & 0.09 \\
\hline Mittelwerte & 1.94 & 2.16 & $\begin{array}{c}0.21^{+} \\
+0.10>\mathrm{P}>0.05\end{array}$ \\
\hline
\end{tabular}

TABElle 5

Brenztraubensäure in Blut

$(\mathrm{mg} / \mathrm{dl})$

\begin{tabular}{|c|c|c|c|}
\hline \multicolumn{4}{|c|}{ Mo-H+Reserpin Gruppe } \\
\hline Tier Nr. & VOR & $\mathrm{NACH}$ & $\mathrm{NACH}-\mathrm{VOR}$ \\
\hline 1 & 2.46 & 4.36 & 1.90 \\
\hline 2 & 2.25 & 4.16 & 1.91 \\
\hline 3 & 2.55 & 4.36 & 1.81 \\
\hline 4 & 2.80 & 4.21 & 1.41 \\
\hline 5 & 2.55 & 5.29 & 2.74 \\
\hline Mittelwerte & 2.52 & 4.48 & $\begin{array}{r}1.95^{++++++} \\
++++++\mathrm{P}<0.001\end{array}$ \\
\hline
\end{tabular}

TABELlE 6

Brenztraubensäure in Blut

(mg/dl)

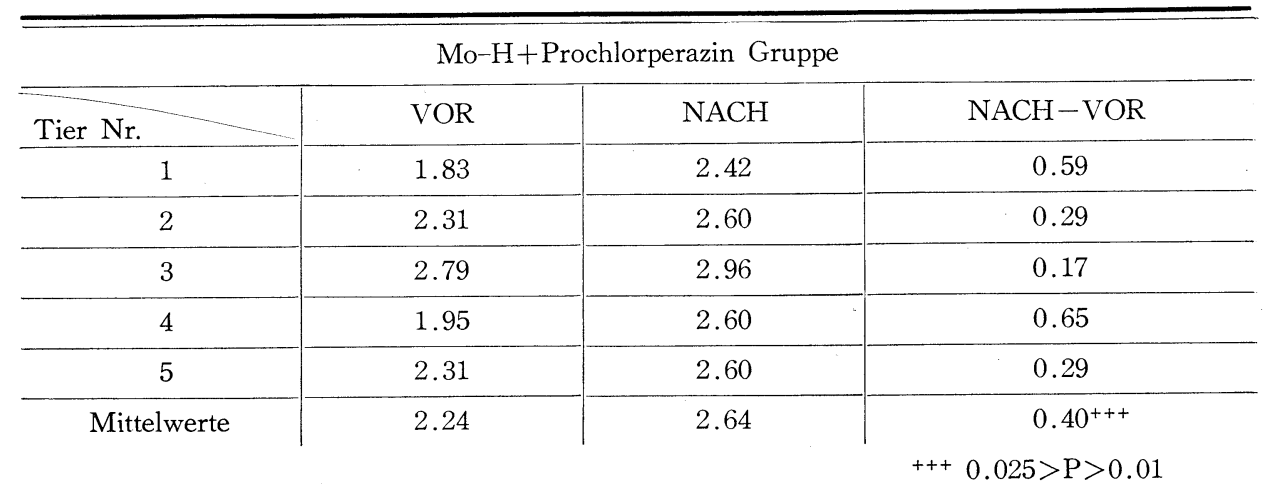


TABELLE 7

Cholesterin in Blut

(mg/dl)

\begin{tabular}{|c|c|c|c|}
\hline \multicolumn{4}{|c|}{ Mo-H Gruppe } \\
\hline Tier $\mathrm{Nr}$. & VOR & $\mathrm{NACH}$ & $\mathrm{NACH}-\mathrm{VOR}$ \\
\hline 1 & 34.98 & 41.85 & 6.87 \\
\hline 2 & 54.19 & 53.91 & -0.28 \\
\hline 3 & 48.12 & 40.94 & -7.18 \\
\hline 4 & 45.09 & 44.58 & -0.51 \\
\hline 5 & 44.08 & 44.85 & 0.77 \\
\hline Mittelwerte & 45.29 & 45.23 & $\begin{array}{l}-0.07^{---} \\
---P>0.50\end{array}$ \\
\hline
\end{tabular}

TABElle 8

Cholesterin in Blut

$(\mathrm{mg} / \mathrm{dl})$

\begin{tabular}{c|c|c|c}
\hline \multicolumn{3}{c}{ Mo-H+Reserpin Gruppe } \\
\hline Tier Nr. & VOR & NACH & NACH-VOR \\
\hline 1 & 63.98 & 89.84 & 25.86 \\
\hline 2 & 58.54 & 86.84 & 28.30 \\
\hline 3 & 57.45 & 67.72 & 10.27 \\
\hline 4 & 52.71 & 69.43 & 16.72 \\
\hline 5 & 76.88 & 100.52 & 23.64 \\
\hline Mittelwerte & 61.91 & 82.87 & $20.96^{+++++}$ \\
& & & $+0.05>\mathrm{P}>0.001$
\end{tabular}

TABElle 9

Cholesterin in Blut

$(\mathrm{mg} / \mathrm{dl})$

\begin{tabular}{c|c|c|c}
\hline \multicolumn{3}{c}{ Mo-H+Prochlorperazin Gruppe } \\
\hline Tier Nr. & VOR & NACH & NACH - VOR \\
\hline 1 & 65.58 & 52.67 & -12.91 \\
\hline 2 & 55.94 & 56.12 & 0.18 \\
\hline 3 & 57.14 & 43.53 & -13.61 \\
\hline 4 & 41.97 & 50.93 & 8.96 \\
\hline 5 & 46.30 & 50.93 & 4.63 \\
\hline Mittelwerte & 53.39 & 50.84 & $-2.55^{---}$ \\
& & & -- P $>0.50$
\end{tabular}




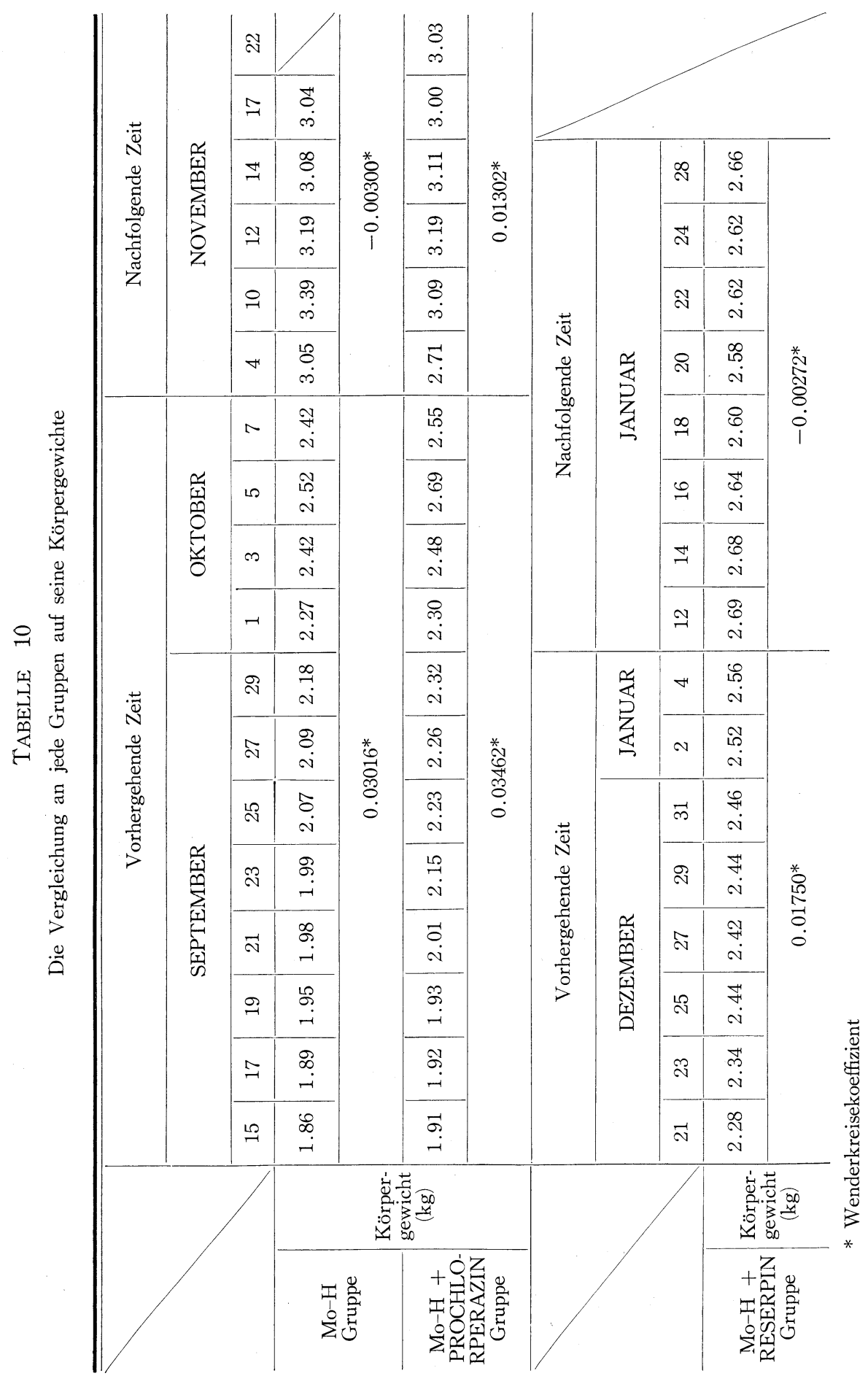


3) Der Einfluss auf Cholesterin in Blut.

Mo-H Gruppe (Tab. 7): Die Differenz war sehr minimal (nicht signifikante Differenz, $\mathrm{p}>0.50$ ).

Mo-H+Reserpin Gruppe (Tab. 8) : Es wurde mit Reserpine nehmt (vor : 61.91 $\mathrm{mg} / \mathrm{dl}$, nach : $82.87 \mathrm{mg} / \mathrm{dl}, 0.005>\mathrm{p}>0.001) \mathrm{zu}$.

Mo-H+Prochlorperazin Gruppe (Tab. 9): Es wurde nicht mit Prochlorperazin nehmt (nicht signifikante Differenz, $\mathrm{p}>0.50$ ) zu.

4) Der Einfluss auf Körpergewicht (Abb. 1, 2, 3, Tab. 10).

Erste waren die $\mathrm{zu}$ administrierter Zeit (nachfolgende Zeit) und nicht verfügter Zeit (vorhergehende Zeit) unterschiedene Wendekreis zwischen genauen Messungen auf Körpergewichten und seinem Tagen gerechnen.

Der Effekt jedes Heilmittels war mit solchen Wandekreis zweien Zeiten dieselben

Abb. 1. Mo-H GRUPPE

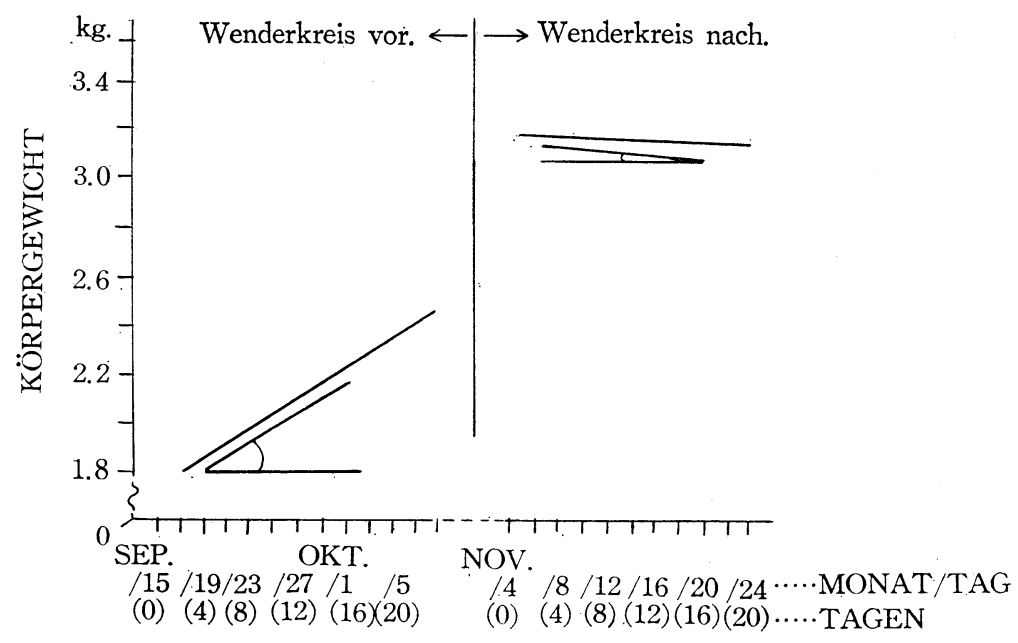

Abb. 2. Mo-H+PROCHLORPERAZIN GRUPPE

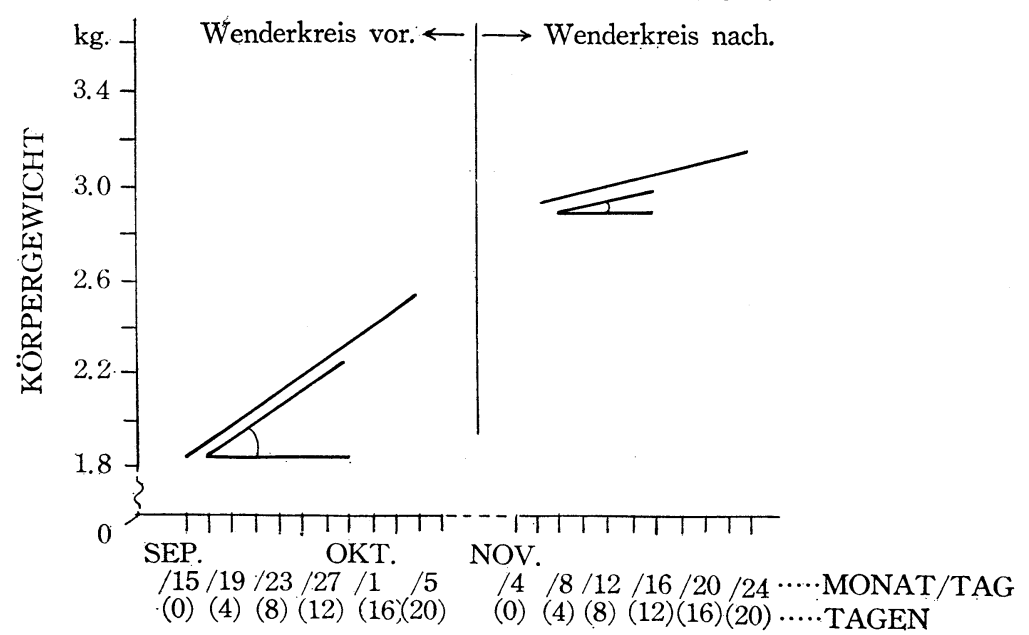


Abb. 3. Mo-H+Reserpin Gruppe

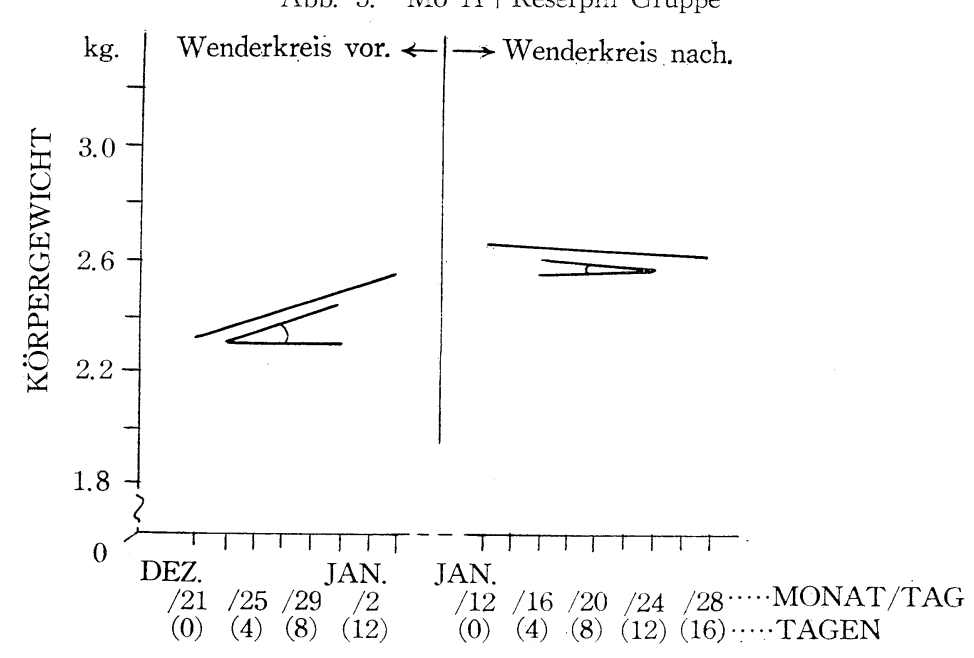

Gruppe vergleicht. Gegen den positive Wenderkreis (positive Wenderkreisekoeffizient) an der vorhergehende Zeit zeigt der negative Wenderkreis (negative Wenderkreisekoeffizient) an der nachfolgende Zeit Inbibition der Gewichtszunahme für den Heilmittel, aber beim seinem positive Wendekreis ist es fragt sich, ob es wahr ist, wenn auch der Koeffizient als an der Vorhergehende weniger gezeigt ist, denn die Gewichtszunahme ist oft nicht deutlicher sogar an der Nachfolgende, die eine Arznei nicht gebrauchen ist, als an der. Vorhergehende, jedoch in solcher Seite nie es schwächt.

Wenn auf diese Weise man jede Gruppen observiert, waren die Schwächungen der Körpergewicht in $\mathrm{Mo}-\mathrm{H}$ Gruppe (vor : 0.03016, nach : -0.00300) und $\mathrm{Mo}-\mathrm{H}+\mathrm{Reserpin}$ Gruppe (vor: 0.01750, nach: -0.00272 ) gefunden.

\section{Diskussion}

Es ist schon aufklärt worden, die Sedation des Reserpin mit der Vorbehandlung des Mo-H zu inhibieren, wenn auch man muss bestreiten, besteht solcher pharmakologische Mechanismus aus Serotonin oder aus Cathecholamin. Unsere diesmalige Untersuchung zeigte, dass die mit Sedationen nach die Rezept des Tranquillizer entspringte Zunahme des Blutzucker Mo-H (Isokarboxazid) inhibiert, der allein es vermindert. gekannt.

Man hat noch nicht den Bericht auf solch einem pharmakologischen Mechanismus

Zweitens, mit dem Zweck, die Beziehung zwischen Steroid Metabolismus und Serotonin zu studieren, prüfte wir Cholesterin in Blut unter Verabreichungen von $\mathrm{Mo}-\mathrm{H}$ sowie die Tranquillizer. Es ist wenigst auch die Vorschung auf dieses Seite noch dazu Report, von welchem Kato Serotonin im Zentrum des mit Kortison administrierten Ratten mass [11].

Der interessante Teil aus diese unsere Experiment war, dass der Stoff, der an Quantität des Cholesterin einfluss, nur Reserpin weder Isokarboxazid noch Prochlorperazin war. 


\section{Zusammenfassung}

Mit dem Zweck, die Relation zwischen Serotonin in das Gehirn und Blutzucker, Brenztraubensäure sowie Cholesterin zu untersuchen, züchte wir Kaninchen auf einige Zeit, indem die Autor subkutane Injektion des Reserpin, Mo-H (Isokarboxazid), und Prochlorperazin machten.

Dabei fanden wir, 1) dass Isokarboxazid, welches Serotonin im Zentrum vermehren, Blutzucker vermindert, aber, wenn diesem Mittel Reserpin des Serotoninfreisetzers und Prochlorperazin des nicht Serotoninfreisetzers kombiniertet, nicht an Quantität es bildeten sich um, ferner 2) dass Isokarboxazid Brenztraubensäure in Blut vergrösserten, die, auch wenn Reserpin oder Prochlorperazin forgertet, nicht vermindert sich, und 3) dass Isokarboxazid die Quantität des Cholesterin in Blut nicht bildeten um, aber, wenn mit Reserpin gingt, es vermehrten sich.

Prof. Dr. M. SATO danken wir für ihre Hilfe bei der Durchführung der Versuche.

\section{Literatur}

[1] M. Kobayashi : Influences with Central Nervous Drugs on Experimental Developments of Dental Caries.; Nihon Univ. dent. J. (Tokyo, Japan), Vol. 38, 123-155, (1964).

[2] Y. Asakura, T. Mori \& K. Suzuki : Experimental Pharmacological Studies on Metabolic Mechanism of Hard Tissues [Report 8]. Influences of Central Nervous Depressants on the Rat Molars, its femur, Serum-Calcium and Blood Sugar; Nihon Univ. dent. J. (Tokyo, Japan), Vol. 39, 184-193 (1965)

[ 3 ] Y. Asakura, J. Inoue, T. Ninoh \& M. Suganuma: Die Einfluss der mit MAOI, Promethazin sowie Atropin Kombinierten Tranquillizer, auf Blutzucker, - cholesterin, -kalzium und Harten Gewebe der Ratte; Jap. J. Oral Biol. (Tokyo, Japan), Vol. 7, 29-30 (1965).

[4] B. B. Brodie \& P. A. SHore: A Concept for a Role of Serotonin and Norepinephrine as Chemical Mediators in the Brain; Annals New York Academy of Science, Vol. 66, 631642 (1957).

[5] B. B. Brodie, S. Spector \& P. A. SHORE: Interaction of Drugs with Norepinephrine in the Brain; Pharmacol. Rev. Vol. 11, 548-566 [1959].

[6] T. Ninnoh, J. Inoue, Y. Asakura, T. Nojiri \& M. Yamamoto: Influences of Tranquilizers Combined with MAOI and Promethazine, on Blood Sugar in Rat. Nihon Univ. dent. J. (Tokyo, Japan), Vol. 39, 329-332 (1965).

[7] Y. Wakamatsu: Die Einfluss der mit MAOI sowie Promethazin Kombinierten Tranquillizer, auf Brenztraubensäure im Blut der Ratte; Unveröffentlichung.

[8] T. OHTA, K. Ishikawa, Y. TAkatori, N. IChibangase, R. TAkahashi \& I. IshiGURO: Das Lehrbuch für Biochemischer Versuch; S. 99-S. 101, HIROKAWA Publizierende Komp., Tokyo, Japan (1962).

[9] M. SAIToH: Die Versuch für Klinische Chemie von Elektrophotometer; S. 168-S. 170, Nanzan Doh Komp., Tokyo, Japan (1955).

[10] Dito, S. 208-S. 209.

[11] R. Kato \& L. Valzelli : Cortisone e 5-idrossitriptamina Cerebrale; Boll. Soc. It. Biol., Sper. 34, 1402-1404 (1958). 